\title{
Correlation of inhibitions to structural aspects of chloroplast proteins: A murburn perspective
}

\author{
Vijay Nirusimhan, Daniel A. Gideon*, Kelath Murali Manoj* \\ *Corresponding author (ORCID: 0000-0003-4515-994X), ${ }^{1}$ Satyamjayatu: The Science \& Ethics Foundation, \\ Kulappully, Shoranur-2 (PO), Palakkad District, Kerala, India-679122. \\ Email: satyamjayatu@yahoo.com
}

\begin{abstract}
In this manuscript, we we first present a brief review of the structural awareness on chloroplasts, the two photosystems (PS I \& PS II, along with the respective light harvesting complexes and chlorophyll binding proteins) and NADPH dehyrogenase complex (NDH). Thereafter, with an in silico approach, we attempt to correlate the photoactive proteins' inhibition by various class of molecules, particularly weedicides. The prevailing understanding relies on topographical-affinity driven binding based explanations for electron transfers and inhibitions. The murburn model of photosynthesis deems DROS (diffusible reactive oxygen species) mediated constructive outcomes as the physiological mechanism for overall outcomes. We unravel key anomalous observations on the inhibitory aspects of the photosynthetic machinery, which were also noted in DROS involving systems like microsomal xenobiotic metabolism and mitochondrial oxidative phosphorylation. Via a comparative logic and applying Ockham's razor, we infer that inhibitory outcomes in several photolysis-photophosphorylation processes are better explained by the murburn model.
\end{abstract}

Keywords: light reaction; inhibition; paraquat; diquat; reactive oxygen species; murburn concept 


\section{Introduction to architectural aspets of chloroplast photosynthetic machinery}

Photosynthesis is the most vital event in photoautotrophs and pertains to the process of light energy-driven (photos) formation of carbohydrates (synthesis). Photosynthesis occurs in chloroplasts, which have a linear dimension of 1 to 10 microns; 5 microns being the average 'size' of these lipid-membrane ensconced organelles with an average width of 2.5 microns. Similar to mitochondria, chloroplasts contain a freely permeable outer membrane and an impermeable inner membrane. A plant cell could have anywhere from one to thousand chloroplasts and each of these plastids has a continuous aqueous core called stroma. Embedded/dispersed in the stroma are found highly stacked self-folded vesicular structures (ranging in 20 to 100 per chloroplast) called grana (with linear dimensions of 500 to $800 \mathrm{~nm}$ ), which are inter-connected through slender membranous lamellae. Each chloroplast contains anywhere from 10 to 100 grana. Each granum may comprise of several thylakoids, which are highly invaginated flattened vesicles with very minute amounts of "aqueous lumen". Thylakoid membranes are 4 to $10 \mathrm{~nm}$ thick and the lumen are 10 to $50 \mathrm{~nm}$ across at the narrow sides. The thylakoids membranes are low on lipids and high in proteins, quite like the inner membrane of mitochondria. However, in the thylakoid membranes, up to $80 \%$ of the lipids belong to the unsaturated galactolipids class (quite unlike phospholipids like cardiolipin that are predominantly found in mitochondria), while only $10 \%$ of the lipids are phospholipids or sulfolipids (sulfoquinovosyl diacylglycerols). The various classes of lipids which confer uniqueness to the thylakoids membrane are known to facilitate the efficient binding and interaction of PSI, PSII, photosytem-LHC and NDH supercomplexes (Sheng et al., 2018). Although the plastid family is comprised of many other members (amyloplasts, chromoplasts, leucoplasts, elaioplasts, gerontoplasts and etioplasts), only green chloroplasts can perform photosynthesis (Kirchhoff, 2019).

A chloroplast may have hundreds thylakoids, and each thylakoid comprises of hundreds or thousands of photosystems and other accompanying proteins/complexes that are required for PlPp pathway. Each photosystem may be multimeric with several tens of protein subunits, along with its dedicated CBPs and LHCs. In toto, the PhotoSystem Core - Chlorophyll Binding Proteins - Light Harvesting Complexes (PSC-CBP-LHC) incorporates hundreds of pigments and 
cofactors belonging to various classes. The various complexes involved in photosynthesis (both cyclic and non-cyclic) are known to exist together as mega/supercomplexes. For example, (Iwai et al. 2010) reported the existence of a large supercomplex containing PSI with its own LHCI, LHCII, Cyt. $b_{6} f$, FNR and PGRL1, an integral membrane protein which essentially was involved in cyclic electron flow (CEF) pathway. In thylakoid membranes, the four most important multiprotein complexes are - PSII-LHCII, PSI-LHCI, cytochrome $b_{6} f$ and ATP synthase. Therefore, a chloroplast would have thousands to millions of copies of various pigment molecules like chlorophylls, carotenoids, xanthophylls (lutein), etc. All pigments are predominantly hydrophobic and characterized by an extended linear or cyclic pi-electron cloud, with alternating single and double bonds. Hence, the spatial arrangement and geometrical placement of various light harvesting pigments around the reaction centers of photosystems I and II is not yet clear. Both Photosystems I \& II are associated with $\sim 200$ chlorophyll and $\sim 50$ carotenoid molecules. An LHC II typically contains $7 \mathrm{Chl} a, 5 \mathrm{Chl} b$ and 2 lutein. Such an LHC may function as a trimer, closely associated with either PS I or PS II. Both PS I and PS II may have several LHC I and LHC II alongside, present in different spatial arrangements, in conjunction with the CBPs. (Herein, it is not deemed necessary to get into the details of the names and positions of the different proteins.) During state transitions, cytochrome $\mathrm{b}_{6} \mathrm{f}$ is known to link PSI and PSII. Generally, most plants have a Chla:Chl $b$ ratio of 2. The PS I / PS II

chlorophyll ratios have been reported to vary from 0.54 to 1.4 ; usually averaging at about 1 . (For more details and specifics on distribution of the pigments in various systems, please refer Caffarri et al., 2014.).

Herein, we investigate the structure-based rationale for inhibitions found in the light reaction of oxygenic photosynthesis. In this regard, we first try to present a structural update on the photosystems. Thereafter, we employ simple in silico docking methodologies and compare the results with known experimental aspects of inhibitions, to make tangible interpretations for the mechanism of light reaction of oxygenic photosynthesis.

\section{Methodology}

Molecular docking of the photosynthesis inhibitors was performed using a protocol similar to the protocol followed in Manoj et al., 2019a. The inhibitor molecules were downloaded from 
PubChem, or obtained by drawing using ChemDraw Ultra 8.0. The receptor proteins, 5XNL (PSII) and 5L8R (PS I) were downloaded from PDB and converted to pdbqt. Using AutoDock Tools (ADT) (Morris et al., 2001), the pdbqt files of ligands and the receptor proteins were prepared. The Gridmap was generated using the AutoGrid. The gridbox parameters were as follows $-72 \times$ $74 \times 72$ xyz points (grid spacing value $0.375 \AA$ ) and the grid center $\mathrm{x}, \mathrm{y}$, and $\mathrm{z}$ were set as 0.728 , 0.918 and 0.860 respectively. The most widely known photosynthesis inhibitors (of PSII and PSI; please see Table 1 for details of molecules chosen) were docked to the subunits reported in literature, where these inhibitors are known to bind. Inhibitory constant ( $k i)$ values and ligand efficiency were retrieved from best-docked poses.

\section{Analysis \& Discussion}

It is first opportune to present a snapshot on the awareness of the structures of the two photosystems (whose component proteins were employed in the docking procedure). Several biophysical methodologies have been used to determine the architecture of PSI and PSII (Golbeck \& van der Est, 2014). While most of the structural insights of the photosystems have been obtained from lower photoautotrophic organisms such as thermophilic cyanobacteria, the exact structural aspects of higher plants remained elusive. The structural biology of PSII has been pursued for decades with the first crystal structure of the PSII dimer of Thermosynechoccus elongatus being solved in 2001 (Zouni et al. 2001). Gradually, more details about the structure of PSII in association with LHCs were deciphered. A major breakthrough came when (Umena et al. 2011) obtained a high resolution (1.9 ̊), from which the atomistic details of the OEC cluster was unambiguously illustrated.

\section{Photosystem II \& PSII-LHCII supercomplex:}

PSII (aka water-plastoquinone oxidoreductase) is a multisubunit protein-cofactor complex containing 20 protein subunits, 80 cofactors (chlorophylls, carotenoids, lipids and ions) and roughly 1000 intrinsic water molecules. It is embedded in the thylakoid membrane of photoautotrophs (cyanobacteria, algae and plants) and its chief role is to oxidize water and reduce plastoquinone. The mature PSII dimer forms super-complexes with a variety of pigment- 
containing antenna proteins (LHC complexes) (Eaton-Rye \& Sobotka, 2017); in the antennae of LHC complex, photons from sunlight excite P680 to P680* and the charge separation which occurs in the antennae lead to formation of a high energy electron which is passed on to reduce plastoquinone. Each photon absorption event then oxidizes the OEC, which after four oxidation events, is known to reset itself by splitting water. Water molecules are photo-oxidized by PSII and the four electrons which are removed from water are transferred to plastoquinone (Schlichting, 2015). The proton gradient which is generated in the process is utilized for ATP synthesis (chemiosmosis) and the electrons are passed on through a series of electron carriers to reduce $\mathrm{NADP}^{+}$to form NADPH. Molecular oxygen is liberated, while water serves as the electron donor. Differences in evolutionary pressures may have led to the variations in the structure and composition of PSII in various photoautotrophs.

Commonly, PSII is composed of subunits D1 and D2 which form the core subunit (containing the P680 RC) and possess the cofactors involved in ETC. Subunits CP47 and CP43 have been shown to act as inner antennae which coordinate majority of the Chla molecules. Apart from the core and the inner antennae protein subunits, other accessory proteins of the complex have lower molecular mass and are their functions have not been fully deciphered (Crepin et al., 2016). While the core subunits in PSII are conserved in prokaryotes and eukaryotic photoautotrophs, the structural heterogeneity of PSII is mostly due to the lower molecular weight subunits. PSII exhibits a great degree of dynamic response to variations in light intensities; acclimation to various light intensities involves structural reorganization of PSII-LHC-II supercomplexes (Albanese et al., 2016). Many workers have noted the similarities in composition and organization of the protein subunits (and their pigments/cofactors) and a more detailed review of the structure-function aspects of the photosystems are discussed at length (Gao et al., 2018). PSI is known to be well protected against photoinhibition; the activity of PSI is photoinhibited in the thylakoid membranes and photoinhibition is mediated by ROS scavenging enzymes such as superoxide dismutase (Lima-Melo et al., 2019). PSI is less affected than PSII by high light because PSI is the terminal electron carrier in chloroplasts and was found to be the major site of ROS production (Takagi et al., 2016). 
Recently, the crystal structure of Pisum sativum (pea) PSII-LHCII complex (Molecular mass of 1468.68 KDa) was solved by Cao et al. (2018 BBA) at $2.7 \AA$ (PDB ID: 5XNL). The $\mathrm{C}_{2} \mathrm{~S}_{2} \mathrm{M}_{2}$ supercomplex of Arabidopsis thaliana had also been crystallographically determined by (Su et al., 2017). Their research showed PSII in association with LHCII to form a supercomplex designated as $\mathrm{C}_{2}-\mathrm{S}_{2}-\mathrm{M}_{2}$ type which contained two LHCII trimers (M-LHCII and S-LHCII). Isolation and study of chloroplasts is complicated and X-ray diffraction was found to damage the photosystem structures. To isolate and study the $\mathrm{C}_{2} \mathrm{~S}_{2} \mathrm{M}_{2}$ supercomplex in its physiological (natural) form, Albanese et al. (2017) employed an isolation strategy and characterized the structure of the complex, which was found to exist in a paired $\mathrm{C}_{2} \mathrm{~S}_{2} \mathrm{M}_{2}$ form. Pisum sativum PSII (PsPSII) is a homodimer containing 28 protein subunits and 203 pigments per monomer, of which, 157 are Chls, 44 carotenoid molecules and 2 pheophytins. Cao et al. reported the presence of 1076 water molecules per PSII monomer, while others report the presence of nearly 2800 water molecules in the entire PsPSII dimer. These water molecules were found to be dispersed all over the supercomplex and some were located in the membrane and most of the waters were deemed to be chlorophyll ligands. The organizational details of PS II-LHC complex is shown in Figures $1 \& 2$.

The core complex consists of 4 membrane embedded subunits D1, D2, CP43 and CP47 and 4 hydrophilic lumenal extrinsic proteins (non-covalently attached)-PsbO, PsbP, PsbQ, PsbTn. CP43 and CP47 are present on either side of the D1/D2 core; CP43 and 47 possess 16 and 13 Chl molecules, respectively and act as light harvesting antennae proteins which transfer the energy to the RC. The 12 other subunits (PsbE, PsbF, PsbH, PsbI, PsbJ, PsbK, PsbL, PsbM, PsbTC, PsbW, PsbX and PsbZ) are intrinsic proteins of low molecular mass and their functions are not fully known (Cao et al., 2018). Also, the 1 CP29 and 1 CP26 monomer and 1 S-LHCII trimer was found to bind to the periphery of PSII core complex and 1 CP24 monomer and $1 \mathrm{M}$-LHCII trimer was found to be associated with the outermost region of the supercomplex. Extrinsic proteins of PSII (PsbO-a protein found in all oxygenic systems, PsbP, PsbQ and PsbR) are known to maintain the stability of the inorganic cubane $\mathrm{Mn}_{4} \mathrm{CaO}_{5}$ cluster (OEC center) and enhance the association of ions such as $\mathrm{Ca}^{2+}$ and $\mathrm{Cl}^{-}$with the $\mathrm{OEC}$ and also optimize oxygen evolution (Roose et al., 2016). When comparing plant and cyanobacterial PSII, Gao et al. (2018) enumerated the number of transmembrane helices, chlorophyll, carotenoids, heme, 
plastoquinone. Plant PSII core (D1, CP47, CP43 and D2) possessed 22 TM helices and the other 21-odd peripheral proteins together contained 13 TM helices. The number of chlorophyll molecules was 4, 16, 13 and 4, respectively (in D1, CP47, CP43 and D2); D1 additionally contained 2 pheophytin residues. The number of carotenoids was 1, 3, 3 and 1 in the four core subunits. One heme residue was found to be jointly associated with PsbE and PsbF. The D1 subunit coordinated the $\mathrm{Mn}_{4} \mathrm{CaO}_{5}$ cluster (OEC) and D2 possessed 1 plastoquinone molecule. Additionally, PSII contained $1 \mathrm{HCO}_{3}{ }^{-}$ion, 1 b-type \& $1 c$-type cytochrome, 1 non-heme iron and $>20$ lipid molecules and a miniomun of $2 \mathrm{Cl}^{-}$ions. Another interesting observation is that among the 35 chlorophylls, the $\mathrm{Mg}$ atom of chlorin rings of 7 chlorophylls are not ligated to aminoacids, but are stabilized by water molecules; around 2 additional water molecules are associated with these 7 Chls to render stability.

In higher plants, the PSII-LHCII supercomplexes reach close to 2,000 KDa molecular weights and the typical arrangement of the peripheral antennae are organized into two layers around the PSII core complex. The inner layer around the core is made up of 2 copies each of CP29, CP 26 and S-LHCII $\left(\mathrm{S}_{2}\right)$ and the dimeric core (PSII) is known as $\mathrm{C}_{2}$. This arrangement of $\mathrm{C}_{2} \mathrm{~S}_{2}$ further binds to outer layer $\mathrm{M}_{2}$ (2 copies of $\mathrm{CP} 24$ and $2 \mathrm{M}-\mathrm{LHCII}$ ) to form the standard structure of $\mathrm{C}_{2} \mathrm{~S}_{2} \mathrm{M}_{2}$ complex which is the predominant PSII supercomplex which is acclimatized to limited light (Cao et al., 2018). Thylakoid membranes were explored for the arrangement of the supercomplexes and either random or ordered arrangements of the supercomplexes and the LHCs was discovered. A comprehensive review of the architecture of chloroplasts and PSII supercomplexes in the thylakoids membranes and a summary of the biophysical methods which explore the arrangement of the supercomplexes is available elsewhere (Kouřil et al., 2018). 


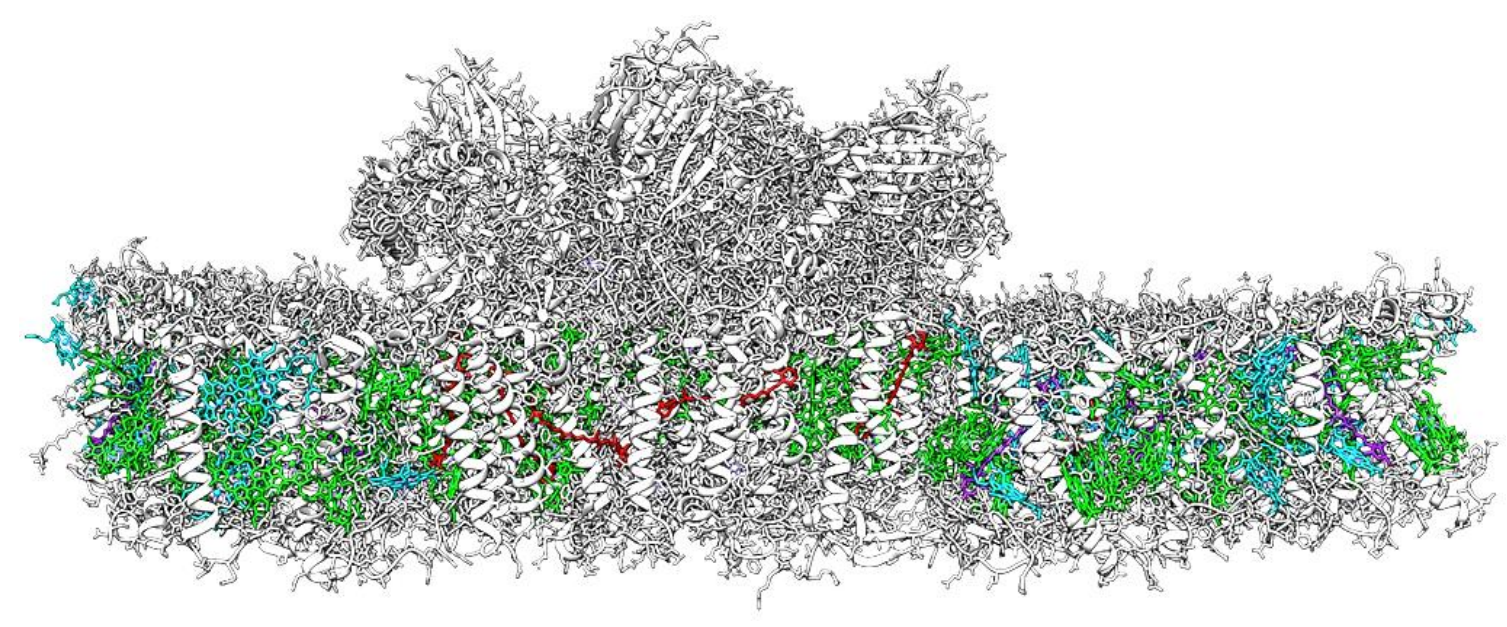

Figure 1: $\mathrm{C}_{2} \mathrm{~S}_{2} \mathrm{M}_{2}$ supercomplex of PSII-LHCII as per the crystal structure of (Su et al., 2017). chla (light green), chlb (cyan), BCR (red) and violoxanthin (violet) in PSII

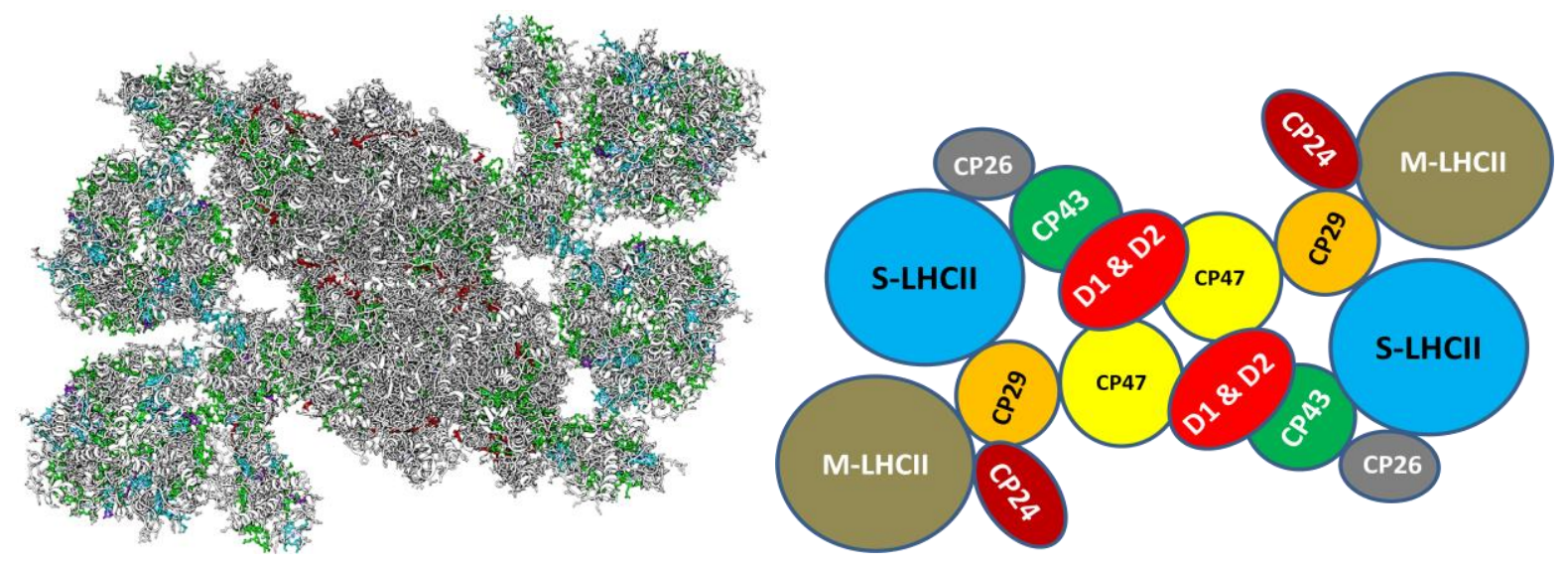

Figure 2: $\mathrm{C}_{2} \mathrm{~S}_{2} \mathrm{M}_{2}$ supercomplex of PSII-LHCII as per the crystal structure of (Su et al., 2017). Chla-green; Chlb-cyan; Car (BCR)-red and violaxanthin-purple. A representative image of the Pisum sativum $\mathrm{C}_{2} \mathrm{~S}_{2} \mathrm{M}_{2}$ supercomplex of PSII-LHCII as per the crystal structure above. D1, CP43, CP47 and D2 subunits constitute the dimeric core of PSII while CP24 and CP29 are peripheral antenna proteins which aid in interaction of S-LHCII and M-LHCII to the PSII core. This is a very simplified version which omits several small polypeptides.

\section{Photosystem I (PSI) and PSI-LHCI supercomplex:}

PSI harnesses solar energy through photosensitive pigments and transfers the electron from plastocyanin, a copper-containing protein to ferredoxin on the other side of the membrane which reduces $\mathrm{NADP}^{+}$to NADPH. PSI is a marvellous photosynthetic supercomplex 'machine' with a 
quantum efficiency of close to unity (despite its astounding complexity), because of the precise orientation of pigments (which is believed to be conserved) and their interactions with the surrounding protein environment (Suga et al., 2016).

Mazor et al. (2015) solved the structure of plant PSI-LHCI supercomplex at $2.8 \AA$ resolution, providing one of the most complete and updated structures of plant PSI-LHCI until now. This supercomplex has PSI subunits at the core region and 4 peripheral LHCI units. The PSI-LHCI supercomplex has a semispherical shape (dimesions of $140 \AA \times 40 \AA$ ) is made up of 16 protein subunits and around 192 light harvesting pigment molecules, 35 lipid molecules, highly conserved FeS clusters and an elaborate water molecule network (Mazor et al., 2015). The 200 odd light harvesting pigments are non-covalently bound to the PSI complex. The While the PSI core is a heterotrimer in cyanobacteria (Hankamer et al., 2001), it is a monomer in higher plants containing upto 16-19 subunits. In P. sativum PSI (PDB ID: 3LW5), there were 17 core subunits, 199 pigment/cofactor molecules (174 Chla, 19 carotenoids, 2 phylloquinones, 3[4Fe4S] clusters and 1 lipid molecule with a total molecular mass of $600 \mathrm{KDa}$. While the entire PSI has 16 subunits (PsaA-PsaL, plus PsaN and PsaO), the four critical protein subunits of the RC are PsaG, PsaH, PsaN and PsaO. LHCI consists of 4 protein Lhca subunits (Lhca1-Lhca4) which form the peripheral light harvesting complex (Mazor et al., 2015). The 200 pigment molecules absorb light energy and transfer it to P700 system's special Chl pair located at the heart of PSI, formed by the PsaA and PsaB heterodimer. The primary electron acceptors- Chl-a (A0), phylloquinone (A1) and FX, a [4Fe4S] cluster are also located in the vicinity of the P700 RC and this heterodimer complex alone was found to contain $\sim 80 \mathrm{Chl}$ molecules which can act as an intrinsic light-harvesting antenna. 2 more [4Fe4S] clusters are bound to PsaC, while all the other subunits are known to act as docking sites; for ferredoxin-PsaC, PsaE and PsaD and for PsaF for plastocyanin binding. While LHCI docking to PSI was found to be mediated by PsaK, PsaG, PsaJ and PsaF, the subunits PsaH, Psal and probably, PsaI were shown to be important for LHCII association with PSI.

Supercomplexes of maize PSI with LHCI and LHCII (PSI-LHCI-LHCII) have been elucidated by Pan et al. (2018) using cryo-EM with a resolution of $3.3 \AA$. While the 16 subunits reported previously in Pea PSI were conserved, the Pan et al. (2018) study revealed the presence of two 
more loosely associated small subunits, PsaO and PsaN which were not identified earlier in the work of Mazor et al. 2017. The two proteins, PsaG and PsaH are found only in higher plants and are absent in cyanobacterial PSI. 1 LHCII trimer was found to bind to PSI near the PsaA subunit side. The two LHC complexes were not coplanar with PSI, but were slightly tilted by $10^{\circ}$, leading to a curved structure. When the maize and pea structures were superimposed, the PSI structures of both these plants were astoundingly similar, with the maize complex containing two small additional subunits ( $\mathrm{PsaO}$ and PsaN) and only minor variations in the hydrophilic loop regions.

\section{A survey and analysis of various types of inhibitors of PI-Pp}

Approximately, 30\% of the commercial herbicides fall under the category of Pl-Pp inhibitors and they are supposed to work by binding to specific loci of photosynthetic proteins, thereby preventing crucial electron transfers. Although inhibitors of Cyt. $b_{6} f$ are known, the majority of commercial inhibitors can be broadly divided into two categories- those that affect PSI and those that affect PSII. In both cases, the physiological symptoms are only seen if plants are illuminated (that is- the inhibitors disrupt the ETC and this process damages the plant) and is found to lead to chlorosis and necrosis, ultimately resulting in plant death. The carotenoids and xanthophylls present in the chloroplasts are also supposed to protect chlorophylls from photo-oxidation (Duke et al., 1993; Ahrens, 1994; Andersson, 1996; Ross \& Lembi, 1999). The PSI inhibitors (called membrane disruptors, electron interceptors, contact herbicides, etc.) supposedly perturb the ETC by diverting electrons from key Fe-S proteins (Fuerst \& Norman, 2017) and are represented by bipyridyliums like paraquat (methyl viologen). The molecule per se is not deemed toxic but it is supposed to lead to the production of DROS, which are deemed to lead to cellular disruption. The PSII inhibitors (represented by triazines, substituted ureas and uracils, phenylcarbamates, nitriles, pyridazines, pyridazinones, acid amides, benzothiadiazoles, etc.) are supposed to bind to the thylakoid D1 $\mathrm{Q}_{\text {в }}$ protein, thereby blocking electron transfers to the mobile plastoquinone pool. (There is yet another physical mode of inhibition, called photoinhibition, which usually results from blue light-induced damage to oxygen-evolving complex accompanying PSII. The mechanism of damage supposedly involves DROS. This is not focused herein.) Besides these 
well-studied inhibitions of Pl-Pp, there are also reports of inhibitions sponsored by histones and polycations, reminiscent of the inhibition of mOxPhos (Brand et al., 1972).

We believe that the mechanism of inhibitions proffered by the various chemicals is poorly understood with the classical binding-based perspective. For select inhibitors (molecular structures are shown in Figure 3), we present a compilation of reported indicators of activity and affinity, and compare them with their blind-docking energies (as shown in Table 1). The protocols adopted were similar to our works reported earlier (Venkatachalam et al., 2016; Manoj et al., 2019). The docked small molecules did not show high affinities, as the $\mathrm{K}_{\mathrm{i}}$ ranged from $10^{-4}$ to $10^{-6} \mathrm{M}$, for the diverse classes of inhibitors. This is when the $\mathrm{EC}_{50}$ values ranged from $10^{-5}$ to $10^{-9} \mathrm{M}$ and when the experimentally determined $\mathrm{K}_{\mathrm{d}}$ values were $\sim 1 \mu \mathrm{M}$ for the four molecules: atrazine, diuron, bromacil and bromoxynil (Zimmermann et al., 2006). Restated, when making a comparison for $\mathrm{EC}_{50}$ values, some of the molecules effectively inhibited the reactions at significantly lower concentration ranges than the in silico $K_{i}$ or experimental $K_{d}$ values. We have solved such conundrums in heme enzymology, mXM and mOxPhos systems by attributing these outcomes to the involvement of DROS-inhibitor interactions (Manoj, 2006; Manoj \& Hager, 2001, 2006; Manoj et al., 2010; Manoj et al., 2016a; Manoj, 2018a, b, c; Manoj et al., 2019a, b). Here, in the Pl-Pp system, we come across curious examples, such as: though atrazine had slightly better binding terms over diuron (in silico $\mathrm{K}_{\mathrm{i}}$ of $100 \mu \mathrm{M}$ and $200 \mu \mathrm{M}$ respectively; experimental $\mathrm{K}_{\mathrm{d}}$ of $600 \mathrm{nM}$ and $800 \mathrm{nM}$ respectively) with PS II, they showed significant differences in $\mathrm{EC}_{50}$ values: $200 \mathrm{nM}$ and $20 \mathrm{nM}$, respectively. Further, molecules containing the sulfur lone pair conjugated to the triazine ring gave better inhibitions (say, S-Me compared to O$\mathrm{Me}$ ) within the same class of triazines, signifying that the key reaction involves an electrophilic species. If binding were to be the most important criterion, the carbamates or pyridate should have given high inhibitions, but such expectations were seen to be unmet by experimental findings. Very importantly, it was seen that the $\mathrm{EC}_{50}$ (or experimental $\mathrm{K}_{\mathrm{i}}$ values) reported for the same molecule varied significantly across research groups and target organisms (Wilski et al., 2006; Wilkinson et al., 2015). This is not expected in a binding-based scenario because the proteins and small molecules are supposedly conserved across species. These observations strongly suggest that it is not binding-based outcomes that determine inhibitions, but it is reaction-mechanism based events that lead to inhibitions by these potent agents. This is 
corroborated by the fact that inhibitions are noted only with photosynthetic physiology (at the impingement of light, and not otherwise); a clear signal that supports the murburn proposal that DROS are the obligatory agents involved in the interactive scheme with these molecules. In this regard, the reactions of molecules like diuoron and paraquat are documented with DROS like hydroxyl or superoxide radicals (da Silva Simões et al., 2017; Manonmani et al., 2020). Therefore, this is strong evidence in support of the murburn perspective of Pl-Pp.
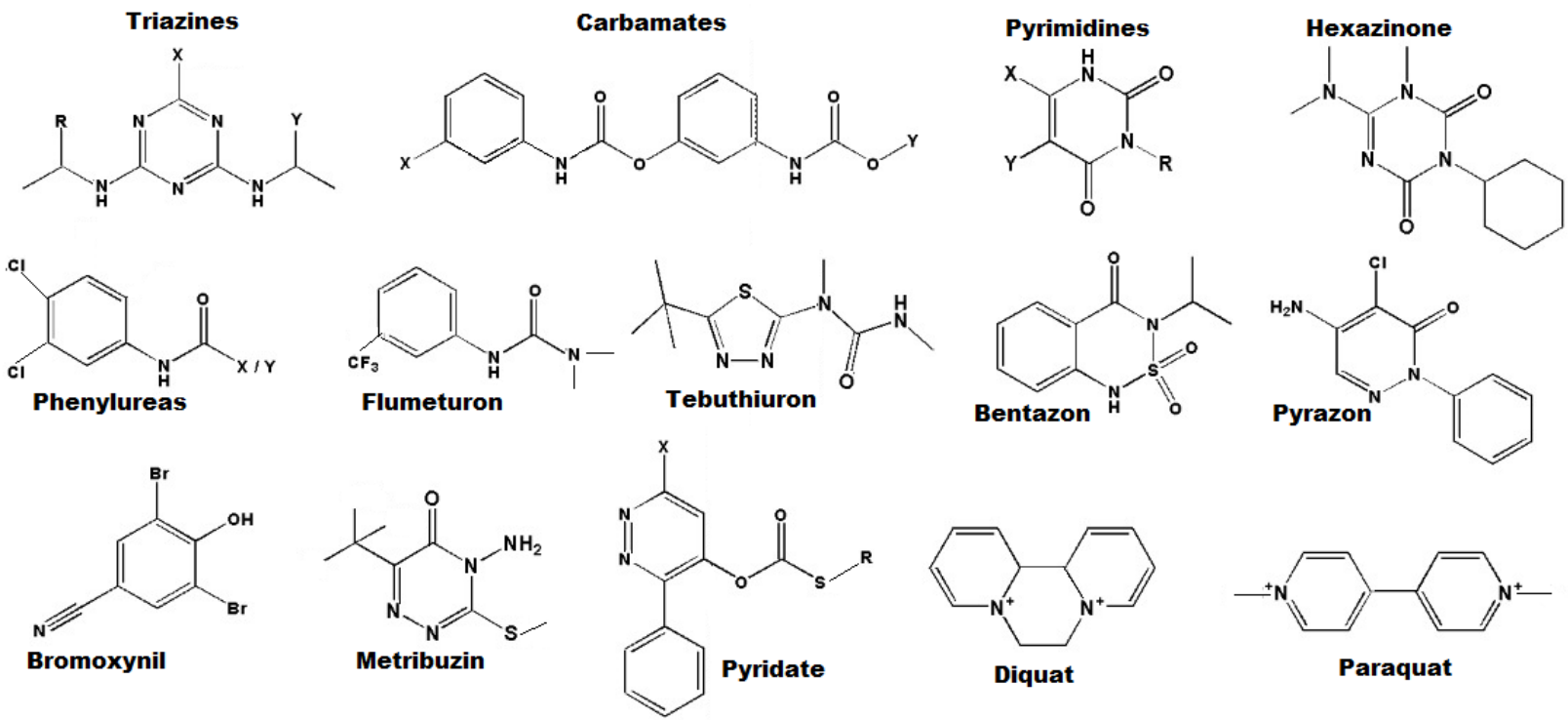

Figure 3: Backbones and structures of some inhibitors of PI-Pp surveyed in the current study.

Table 1: Comparison of in silico $\mathrm{K}_{\mathrm{i}}$ values and experimental $\mathrm{EC}_{50}$ of some inhibitors of photosystems I \& II.

\begin{tabular}{|l|c|c|c|c|c|}
\hline No & Class & $\begin{array}{c}\text { Name / Substitutions } \\
(\mathbf{R}, \mathbf{X}, \mathbf{Y})\end{array}$ & $\begin{array}{c}\text { Binding energy } \\
(\mathbf{k c a l} / \mathbf{m o l})\end{array}$ & $\begin{array}{c}\text { Predicted } \boldsymbol{K}_{\mathbf{i}} \\
(\mathbf{M})\end{array}$ & $\begin{array}{c}\text { Experimental } \\
\boldsymbol{E} \boldsymbol{C}_{\mathbf{5 0}}(\mathbf{M}),(\mathbf{r e f})\end{array}$ \\
\hline \multicolumn{5}{|c|}{$\mathbf{5 X N L}-\mathbf{P S}$ II, D1 } \\
\hline 01 & Triazines & Simazine (H,Cl,H) & -4.7 & $4 \times 10^{-4}$ & $1 \times 10^{-7(1)}$ \\
\hline 02 & Triazines & Atrazine (Me,Cl,H) & -5.1 & $2 \times 10^{-4}$ & $2 \times 10^{-7(1)}$ \\
\hline 04 & Triazines & Propazine (Me,Cl,Me) & -5.5 & $1 \times 10^{-4}$ & $3 \times 10^{-7(2)}$ \\
\hline 05 & Triazines & $\begin{array}{c}\text { Prometon (Me,OMe, } \\
\text { Me) }\end{array}$ & -5.1 & $2 \times 10^{-4}$ & $5 \times 10^{-7(2)}$ \\
\hline 06 & Triazines & Ametryn (H,SMe,Me) & -5.0 & $2 \times 10^{-4}$ & $2 \times 10^{-8(1)}$ \\
\hline
\end{tabular}




\begin{tabular}{|c|c|c|c|c|c|}
\hline & & (Me,SMe,Me) & & & \\
\hline 07 & Carbamates & Desmedipham $(\mathrm{H}, \mathrm{Me})$ & -6.2 & $3 \times 10^{-5}$ & $1 \times 10^{-6(3)}$ \\
\hline 08 & Carbamates & Phenmedipham (Me,Et) & -6.6 & $1 \times 10^{-5}$ & $1 \times 10^{-6(3)}$ \\
\hline 09 & Pyrimidines & Bromacil (s-Bu,Me,Br) & -5.7 & $6 \times 10^{-5}$ & $1 \times 10^{-7(1)}$ \\
\hline 10 & Pyrimidines & Terbacil (t-Bu,Me,Cl) & -5.8 & $5 \times 10^{-5}$ & $6 \times 10^{-7(4)}$ \\
\hline 11 & Phenyl ureas & Propanil (Et) & -5.7 & $6 \times 10^{-5}$ & $2 \times 10^{-9(5)}$ \\
\hline 12 & Phenyl ureas & Diuron $\left(\mathrm{N}(\mathrm{Me})_{2}\right)$ & -5.4 & $1 \times 10^{-4}$ & $2 \times 10^{-8(1)}$ \\
\hline 13 & Phenyl ureas & Linuron $(\mathrm{N}(\mathrm{Me})(\mathrm{OMe}))$ & -5.3 & $1 \times 10^{-4}$ & $5 \times 10^{-8(6)}$ \\
\hline 14 & Other ureas & Fluometuron & -5.1 & $2 \times 10^{-4}$ & $6 \times 10^{-7(1)}$ \\
\hline 15 & Other ureas & Tebuthiuron & -5.7 & $7 \times 10^{-5}$ & $1 \times 10^{-7(1)}$ \\
\hline 16 & Miscellaneous & Bromoxynil & -5.4 & $1 \times 10^{-4}$ & $9 \times 10^{-6(7)}$ \\
\hline 17 & Miscellaneous & Bentazone & -6.0 & $4 \times 10^{-5}$ & $1 \times 10^{-5(8)}$ \\
\hline 18 & Miscellaneous & Pyridate (Oct) & -7.5 & $3 \times 10^{-6}$ & $1 \times 10^{-6(9)}$ \\
\hline 19 & Miscellaneous & Hexazinone & -6.6 & $1 \times 10^{-5}$ & $6 \times 10^{-8(1)}$ \\
\hline 20 & Miscellaneous & Metribuzin & -5.5 & $9 \times 10^{-5}$ & $3 \times 10^{-8(1)}$ \\
\hline 21 & Miscellaneous & Pyrazon & -5.5 & $9 \times 10^{-5}$ & $5 \times 10^{-5(10)}$ \\
\hline \multicolumn{6}{|c|}{ 5L8R - PS I, Psa C/D } \\
\hline 22 & Bipyridinium & Diquat & -5.0 & $2 \times 10^{-4}$ & $3 \times 10^{-5(11)}$ \\
\hline 23 & Bipyridinium & Paraquat & -4.5 & $5 \times 10^{-4}$ & $8 \times 10^{-5(12)}$ \\
\hline
\end{tabular}

The $E C_{50}$ values entered in italics are from the same research group, and therefore can be good for a relative comparison within a given inhibitor class or cutting across classes. References: (1) Wilkinson et al. 2015; (2) Faust et al. 2001; (3) Traoré et al. 2018; (4) Peck et al. 2019; (5) Villarroel et al. 2003; (6) Snel et al. 1998; (7) Bettiol et al. 2016; (8) Michel et al. 2004; (9) Gressel et al. 1992; (10) Bisewska et al. 2012; (11) Malaspina et al. 2017; and (12) Qian et al. 2009

Placing the wide topographical profiles of the diverse inhibitory molecules with respect to an active site binding ability is not feasible in the context of the inhibitions seen for the two photosystems. With the classical view, it is inexplicable as to why cyanide could serve as a toxic principle in the Pl-Pp process. Both Photosystems I and II (whose functioning is supposedly inhibited by cyanide) are not known to get inhibited owing to any hemeFe binding-based effects, either. Emerson showed that the dark process is temperature sensitive but the light process is not (with respect to oxygen yield). At low temperature, $10 \mu \mathrm{M}$ cyanide gave higher inhibition at short duration of darkness or continuous light, whereas it gave no inhibitory effect at longer dark periods. This could signify that light stimulation is associated with DROS production and DROS 
has utility in the photosynthetic process, which is subverted by CN. Such outcomes can be reasoned out within the murburn scheme, as cyanide modulates DROS (Parashar et al., 2014; Manoj et al., 2016b; Manoj, 2020; Manoj et al., 2020).

\section{Conclusions}

With respect to the murburn model of Pl-Pp (photolysis-photophosphorylation), we have argued earlier that the inhibitions proffered by cyanide and the effects of uncouplers in the bioenergetic systems are better explained by the murburn model (Manoj \& Soman, 2020; Gideon et al., 2020; Manoj et al., 2020a-d; Manoj \& Manekkathodi, 2021). Here, we presented elementary support for the murburn mechanistic model of inhibitions in the light reaction of photosynthesis, which are based on the outcomes of the mechanism-based DROS interactions, and not primarily as an outcome of the binding of an inhibitor (weedicide) at a particular locus on the photosynthetic protein.

\section{References}

Ahrens W.H., Herbicide Handbook ; Seventh Edition, Weed Science Society of America, 1994.

Albanese, P., Nield, J., Tabares, J. A. M., Chiodoni, A., Manfredi, M., Gosetti, F., ... \& Pagliano, C. (2016). Isolation of novel PSII-LHCII megacomplexes from pea plants characterized by a combination of proteomics and electron microscopy. Photosynthesis research, 130(1), 19-31.

Andersson, B., \& Barber, J. (1996). Mechanisms of photodamage and protein degradation during photoinhibition of photosystem II. In Photosynthesis and the Environment (pp. 101-121). Springer, Dordrecht.

Bettiol, C., De Vettori, S., Minervini, G., Zuccon, E., Marchetto, D., Ghirardini, A.V., Argese, E. Assessment of phenolic herbicide toxicity and mode of action by different assays, Environmental Science and Pollution Research, 23 (2016) 7398-7408.

Bisewska, J., Sarnowska, E.I., Tukaj, Z.H. Phytotoxicity and antioxidative enzymes of green microalga (Desmodesmus subspicatus) and duckweed (Lemna minor) exposed to herbicides MCPA, chloridazon and their mixtures, Journal of Environmental Science and Health, Part B, 47 (2012) 814-822.

Brand, J., Baszynski, T., Crane, F.L., Krogmann, D.W. Selective inhibition of photosynthetic reactions by polycations, The Journal of biological chemistry, 247 (1972) 2814-2819.

Caffarri S., Tibiletti T., Jennings, R.C. Santabarbara, S. A comparison between plant photosystem I and photosystem II architecture and functioning, Current protein \& peptide science, 15 (2014) 296-331.

Cao, P., Su, X., Pan, X., Liu, Z., Chang, W., \& Li, M. (2018). Structure, assembly and energy transfer of plant photosystem II supercomplex. Biochimica et Biophysica Acta (BBA)-Bioenergetics, 1859(9), 633644. 
Crepin, A., Santabarbara, S., \& Caffarri, S. (2016). Biochemical and spectroscopic characterization of highly stable photosystem ii supercomplexes from Arabidopsis. Journal of Biological Chemistry, 291(36), 19157-19171.

da Silva Simões, M., Bracht, L., Parizotto, A.V., Comar, J.F., Peralta, R.M., Bracht, A. The metabolic effects of diuron in the rat liver, Environmental Toxicology and Pharmacology, 54 (2017) 53-61.

Devine, M., Duke, S. O., \& Fedtke, C. (1992). Physiology of Herbicide Action. PTR Prentice Hall.

Eaton-Rye, J. J., \& Sobotka, R. (2017). Assembly of the photosystem II membrane-protein complex of oxygenic photosynthesis. Frontiers in plant science, 8, 884 .

Faust, M., Altenburger, R., Backhaus, T., Blanck, H., Boedeker, W., Gramatica, P., Hamer, V., Scholze, M., Vighi, M., Grimme, L.H. Predicting the joint algal toxicity of multi-component s-triazine mixtures at low-effect concentrations of individual toxicants, Aquatic Toxicology, 56 (2001) 13-32.

Fuerst E.P., Norman M.A., Interactions of Herbicides with Photosynthetic Electron Transport, Weed Science, 39 (2017) 458-464.

Gao, J., Wang, H., Yuan, Q., \& Feng, Y. (2018). Structure and function of the photosystem supercomplexes. Frontiers in plant science, 9, 357.

Golbeck, J. H., \& van der Est, A. (2014). The biophysics of photosynthesis. Springer New York.

Gressel, J., Evron, Y. Pyridate is not a two-site inhibitor, and may be more prone to evolution of resistance than other phenolic herbicides, Pesticide Biochemistry and Physiology, 44 (1992) 140-146.

Hankamer, B., Morris, E., Nield, J., Carne, A., \& Barber, J. (2001). Subunit positioning and transmembrane helix organisation in the core dimer of photosystem II. FEBS letters, 504(3), 142-151.

Iwai, M., Takizawa, K., Tokutsu, R., Okamuro, A., Takahashi, Y., \& Minagawa, J. (2010). Isolation of the elusive supercomplex that drives cyclic electron flow in photosynthesis. Nature, 464(7292), 12101213.

Kirchhoff, H. (2019). Chloroplast ultrastructure in plants. New Phytologist, 223(2), 565-574.

Kouřil, R., Nosek, L., Semchonok, D., Boekema, E. J., \& Ilík, P. (2018). Organization of plant photosystem II and photosystem I supercomplexes. In Membrane Protein Complexes: Structure and Function (pp. 259-286). Springer, Singapore.

Lima-Melo, Y., Alencar, V. T., Lobo, A. K., Sousa, R. H., Tikkanen, M., Aro, E. M., ... \& Gollan, P. J. (2019). Photoinhibition of photosystem I provides oxidative protection during imbalanced photosynthetic electron transport in Arabidopsis thaliana. Frontiers in plant science, 10, 916.

Malaspina, I.C., Cruz, C., Garlich, N., Bianco, S., Pitelli, R.A., Effectiveness of diquat, both isolated and associated with copper sources in controlling the hydrilla verticillata submerged macrophytes and ankistrodesmus Gracilis microphyte, Planta Daninha, 35 (2017).

Manoj, K.M. Aerobic Respiration: Criticism of the Proton-centric Explanation Involving Rotary Adenosine Triphosphate Synthesis, Chemiosmosis Principle, Proton Pumps and Electron Transport Chain, Biochem Insights, 11 (2018c) 1178626418818442.

Manoj, K.M. Chlorinations catalyzed by chloroperoxidase occur via diffusible intermediate(s) and the reaction components play multiple roles in the overall process, Biochimica et Biophysica Acta (BBA) Proteins and Proteomics, 1764 (2006) 1325-1339.

Manoj, K.M., Aerobic Respiration: Criticism of the Proton-centric Explanation Involving Rotary Adenosine Triphosphate Synthesis, Chemiosmosis Principle, Proton Pumps and Electron Transport Chain, Biochem Insights, 11 (2018b) 1178626418818442. 
Manoj, K.M., Gade, S.K., Mathew, L. Cytochrome P450 reductase: a harbinger of diffusible reduced oxygen species, PLoS One, 5 (2010) e13272.

Manoj, K.M., Gade, S.K., Venkatachalam, A., Gideon, D.A. Electron transfer amongst flavo- and hemoproteins: diffusible species effect the relay processes, not protein-protein binding, RSC Advances, 6 (2016a) 24121-24129.

Manoj, K.M., Hager, L.P. A colorimetric method for detection and quantification of chlorinating activity of hemeperoxidases, Analytical Biochemistry, 348 (2006) 84-86.

Manoj, K.M., Hager, L.P., Utilization of peroxide and its relevance in oxygen insertion reactions catalyzed by chloroperoxidase, Biochimica et Biophysica Acta (BBA) - Protein Structure and Molecular Enzymology, 1547 (2001) 408-417.

Manoj, K.M., Parashar, A., David Jacob, V., Ramasamy, S. Aerobic respiration: proof of concept for the oxygen-centric murburn perspective, Journal of Biomolecular Structure and Dynamics, 37 (2019a) 45424556.

Manoj, K.M., Parashar, A., Venkatachalam, A., Goyal, S., Satyalipsu, M., Singh, P.G., Gade, S.K., Periyasami, K., Jacob, R.S., Sardar, D., Singh, S., Kumar, R., Gideon, D.A. Atypical profiles and modulations of heme-enzymes catalyzed outcomes by low amounts of diverse additives suggest diffusible radicals' obligatory involvement in such redox reactions, Biochimie, 125 (2016b) 91-111.

Manoj, K.M., Ramasamy, S., Parashar, A., Gideon, D.A., Soman, V., Jacob, V.D., Pakshirajan, K. Acute toxicity of cyanide in aerobic respiration: Theoretical and experimental support for murburn explanation, Biomolecular concepts, 11 (2020) 32-56.

Manoj, K.M., Soman, V. Classical and murburn explanations for acute toxicity of cyanide in aerobic respiration: A personal perspective, Toxicology, 432 (2020) 152369.

Manoj, K.M., Soman, V., David Jacob, V., Parashar, A., Gideon, D.A., Kumar, M., Manekkathodi, A., Ramasamy, S., Pakshirajan, K., Bazhin, N.M. Chemiosmotic and murburn explanations for aerobic respiration: Predictive capabilities, structure-function correlations and chemico-physical logic, Archives of Biochemistry and Biophysics, 676 (2019b) 108128.

Manoj, K.M., The ubiquitous biochemical logic of murburn concept, 2018, 29 (2018a).

Gideon DA, Nirusimhan V, and Manoj KM. (2020). Are plastocyanin and ferredoxin specific electron carriers or generic redox capacitors? Classical and murburn perspectives on two photosynthetic proteins. Journal of Biomolecular Structure and Dynamics. doi: 10.1080/07391102.2020.1835715

Manoj KM, Gideon DA, Jacob VD, and Manekkathodi A. (2020a). Is Z-scheme a tenable explanation for the light reaction of oxygenic photosynthesis? OSF Preprints. doi: 10.31219/osf.io/v6tdf

Manoj KM, Gideon DA, and Parashar A. (2020b). What is the Role of Lipid Membrane-embedded Quinones in Mitochondria and Chloroplasts? Chemiosmotic Q-cycle versus Murburn Reaction Perspective. Cell Biochemistry and Biophysics 79:3-10.

Manoj KM, Gideon DA, Parashar A, and Manekkathodi A . (2020c). Role of thylakoid membranes in oxygenic photosynthesis: A comparative perspective using murburn concept. OSF Preprints. doi: 10.31219/osf.io/8p2sx

Manoj KM, Bazhin NM, Abhinav Parashar, Daniel Gideon, Vivian David Jacob, Afsal Manekkathodi. (2020d). Murburn precepts for the light reaction of oxygenic photosynthesis. OSF Preprints. doi: 10.31219/osf.io/95brg

Manoj KM and Manekkathodi A. (2021). Light's interaction with pigments in chloroplasts: The murburn perspective. Journal of Photochemistry and Photobiology 5: 100015. 
Manonmani, G., Sandhiya, L., Senthilkumar, K. Mechanism and kinetics of diuron oxidation by hydroxyl radical addition reaction, Environ Sci Pollut Res Int, 27 (2020) 12080-12095.

Mazor, Y., Borovikova, A., \& Nelson, N. (2015). The structure of plant photosystem I super-complex at 2.8 A resolution. elife, 4, e07433.

Michel, A., Johnson, R.D., Duke, S.O., Scheffler, B.E. Dose-response relationships between herbicides with different modes of action and growth of Lemna paucicostata: an improved ecotoxicological method, Environ Toxicol Chem, 23 (2004) 1074-1079.

Morris, G. M., Goodsell, D. S., Huey, R., Hart, W. E., Halliday, S., Belew, R., \& Olson, A. J. (2001). AutoDock. Automated docking of flexible ligands to receptor-User Guide.

Pan, X., Ma, J., Su, X., Cao, P., Chang, W., Liu, Z., ... \& Li, M. (2018). Structure of the maize photosystem I supercomplex with light-harvesting complexes I and II. Science, 360(6393), 1109-1113.

Parashar, A., Venkatachalam, A., Gideon, D.A., Manoj, K.M. Cyanide does more to inhibit heme enzymes, than merely serving as an active-site ligand, Biochemical and Biophysical Research Communications, 455 (2014) 190-193.

Peck, C., Corbin, M., Chief, B., ERB VI. Ecological Risk Assessment for the Registration Review of Terbacil., (2019).

Qian, H., Chen, W., Sun, L., Jin, Y., Liu, W., Fu, Z. Inhibitory effects of paraquat on photosynthesis and the response to oxidative stress in Chlorella vulgaris, Ecotoxicology, 18 (2009) 537-543.

Roose, J. L., Frankel, L. K., Mummadisetti, M. P., \& Bricker, T. M. (2016). The extrinsic proteins of photosystem II: update. Planta, 243(4), 889-908.

Ross, M. A., Lembi, C. A., Stewart, C., Stagman, J., \& Carnis, M. (1999). Herbicide incorporation techniques and equipment. Applied Weed Science. 2nd ed. Upper Saddle River, NJ: Prentice-Hall, 371375.

Sheng, X., Liu, X., Cao, P., Li, M., \& Liu, Z. (2018). Structural roles of lipid molecules in the assembly of plant PSII- LHCII supercomplex. Biophysics reports, 4(4), 189-203.

Snel, J.F.H., Vos, J.H., Gylstra, R., Brock, T.C.M. Inhibition of photosystem II (PSII) electron transport as a convenient endpoint to assess stress of the herbicide linuron on freshwater plants, Aquatic Ecology, 32 (1998) 113-123.

Su, X., Ma, J., Wei, X., Cao, P., Zhu, D., Chang, W., ... \& Li, M. (2017). Structure and assembly mechanism of plant C2S2M2-type PSII-LHCII supercomplex. Science, 357(6353), 815-820.

Takagi, D., Takumi, S., Hashiguchi, M., Sejima, T., \& Miyake, C. (2016). Superoxide and singlet oxygen produced within the thylakoid membranes both cause photosystem I photoinhibition. Plant Physiology, 171(3), 1626-1634.

Traoré, H., Crouzet, O., Mamy, L., Sireyjol, C., Rossard, V., Servien, R., Latrille, E., Martin-Laurent, F., Patureau, D., Benoit, P. Clustering pesticides according to their molecular properties, fate, and effects by considering additional ecotoxicological parameters in the TyPol method, Environmental Science and Pollution Research, 25 (2018) 4728-4738.

Umena, Y., Kawakami, K., Shen, J. R., \& Kamiya, N. (2011). Crystal structure of oxygen-evolving photosystem II at a resolution of $1.9 \AA$ A. Nature, 473(7345), 55-60.

Venkatachalam, A., Parashar, A., Manoj, K.M. Functioning of drug-metabolizing microsomal cytochrome P450s: In silico probing of proteins suggests that the distal heme 'active site' pocket plays a relatively 'passive role' in some enzyme-substrate interactions, In Silico Pharmacol, 4 (2016) 2. 
Villarroel, M.J., Sancho E., Ferrando M.D., Andreu E. Acute, chronic and sublethal effects of the herbicide propanil on Daphnia magna, Chemosphere, 53 (2003) 857-864.

Wilkinson, A.D., Collier, C.J., Flores, F., Negri, A.P. Acute and additive toxicity of ten photosystem-II herbicides to seagrass, Scientific reports, 5 (2015) 17443.

Wilski, S., Johanningmeier, U., Hertel, S., Oettmeier, W. Herbicide binding in various mutants of the photosystem II D1 protein of Chlamydomonas reinhardtii, Pesticide Biochemistry and Physiology, 84 (2006) 157-164.

Zimmermann, K., Heck, M., Frank, J., Kern, J., Vass, I., Zouni, A. Herbicide binding and thermal stability of photosystem II isolated from Thermosynechococcus elongatus, Biochimica et Biophysica Acta (BBA) - Bioenergetics, 1757 (2006) 106-114.

Zouni, A., Witt, H. T., Kern, J., Fromme, P., Krauss, N., Saenger, W., \& Orth, P. (2001). Crystal structure of photosystem II from Synechococcus elongatus at $3.8 \AA$ resolution. Nature, 409(6821), 739-743. 